\title{
VARIATIONAL PROBLEMS WITH POINTWISE CONSTRAINTS AND DEGENERATION IN VARIABLE DOMAINS
}

\author{
Alexander A. Kovalevsky and Olga A. Rudakova
}

\begin{abstract}
In this article we deal with a sequence of functionals defined on weighted Sobolev spaces. The spaces are associated with a sequence of domains $\Omega_{s}$ contained in a bounded domain $\Omega$ of $\mathbb{R}^{n}$. The main structural components of the functionals are integral functionals whose integrands satisfy a growth and coercivity condition with a weight and additional terms $\psi_{s} \in L^{1}\left(\Omega_{s}\right)$. For the given functionals we consider variational problems with sets of constraints for functions $v$ of the kind $h(x, v(x)) \leqslant 0$ a. e. in $\Omega_{s}$, where $h: \Omega \times \mathbb{R} \rightarrow \mathbb{R}$. We establish conditions on $h$ and $\psi_{s}$ and on the given domains, weighted spaces and functionals under which solutions of the variational problems under consideration converge in a certain sense to a solution of a limit variational problem with the set of constraints defined by the same function $h$.
\end{abstract}

Mathematics subject classification (2000): 49J45, 49J40, 35B27.

Keywords and phrases: variational problem, integral functional, degenerate integrand, pointwise constraint, variable domains, convergence of minimizers, $\Gamma$-convergence.

\section{REFERENCES}

[1] H. ATtouch AND C. PICARD, Variational inequalities with varying obstacles: the general form of the limit problem, J. Funct. Anal., 50, 3 (1983), 329-386.

[2] L. Boccardo And F. Murat, Homogenization of nonlinear unilateral problems, Composite Media and Homogenization Theory, Progr. Nonlinear Differential Equations Appl., 5, Birkhäuser, Boston, (1991), 81-105.

[3] L. CARbone And R. De ArCangelis, Unbounded functionals: applications to the homogenization of gradient constrained problems, Ricerche Mat., 48, 1 (1999), 139-182.

[4] L. CARbone And R. De ArCangelis, Unbounded Functionals in the Calculus of Variations, Chapman \& Hall/CRC, Boca Raton, 2002.

[5] G. R. Cirmi AND M. M. PorZIO, $L^{\infty}$-solutions for some nonlinear degenerate elliptic and parabolic equations, Ann. Mat. Pura Appl. (4), 169 (1995), 67-86.

[6] G. Dal MASO, Asymptotic behaviour of minimum problems with bilateral obstacles, Ann. Mat. Pura Appl. (4), 129 (1981), 327-366.

[7] G. DAL MASO, Limits of minimum problems for general integral functionals with unilateral obstacles, Atti Accad. Naz. Lincei Rend. Cl. Sci. Fis. Mat. Natur. (8), 74, 2 (1983), 55-61.

[8] G. Dal Maso And A. Defranceschi, Convergence of unilateral problems for monotone operators, J. Analyse Math., 53 (1989), 269-289.

[9] G. Dal MASO, An Introduction to $\Gamma$-convergence, Birkhäuser, Boston, 1993.

[10] R. De ARCANGelis, Compactness and convergence of minimum points for a class of nonlinear nonequicoercive functionals, Nonlinear Anal., Theory Methods Appl., 15, 4 (1990), 363-380.

[11] R. De ArCangelis And P. Donato, Homogenization in weighted Sobolev spaces, Ricerche Mat., 34, 2 (1985), 289-308.

[12] R. De ArCangelis and F. Serra Cassano, On the homogenization of degenerate elliptic equations in divergence form, J. Math. Pures Appl. (9), 71, 2 (1992), 119-138.

[13] R. De ARCANGElis AND F. SERRA CASSANO, On the convergence of solutions of degenerate elliptic equations in divergence form, Ann. Mat. Pura Appl. (4), 167 (1994), 1-23. 
[14] V. DE CiCco, Limits of nonlinear weighted Dirichlet problems in varying domains, Nonlinear Anal. Ser. A: Theory Methods, 48, 2 (2002), 274-270.

[15] E. De Giorgi AND T. Franzoni, Su un tipo di convergenza variazionale, Atti Accad. Naz. Lincei Rend. Cl. Sci. Fis. Mat. Natur. (8), 58, 6 (1975), 842-850.

[16] A. Gaudiello, Sulla convergenza dei punti di minimo di funzionali degeneri non equicoercitivi, Rend. Accad. Sci. Fis. Mat. Napoli (4), 57 (1990), 125- 168.

[17] D. Gilbarg and N. S. Trudinger, Elliptic Partial Differential Equations of Second Order, Springer-Verlag, Berlin, 1983.

[18] F. GUGLIELMINO AND F. NiCOLOSI, $W$-solutions of boundary value problems for degenerate elliptic operators, Ricerche Mat., 36 (1987), 59-72.

[19] J. Heinonen, T. Kilpeläinen And O. Martio, Nonlinear Potential Theory of Degenerate Elliptic Equations, The Clarendon Press, Oxford University Press, New York, 1993.

[20] E. YA. KHRUSLOV, Asymptotic behavior of the solutions of the second boundary value problem in the case of the refinement of the boundary of the domain, Mat. Sb., 106, 4 (1978), 604-621.

[21] A. A. KovALEVSKII, On the connectedness of subsets of Sobolev spaces and the $\Gamma$-convergence of functionals with varying domain of definition, Nelinejnye granichnye zadachi, 1 (1989), 48-54.

[22] A. A. Kovalevs KII, Some problems connected with the problem of averaging variational problems for functionals with a variable domain, Current Analysis and its Applications, Naukova Dumka, Kiev, (1989), 62-70.

[23] A. A. KovaleVSKII, $G$-convergence and homogenization of nonlinear elliptic operators in divergence form with variable domain, Russian Acad. Sci. Izv. Math., 44, 3 (1995), 431-460.

[24] A. A. KovalevSKiI, On the $\Gamma$-convergence of integral functionals defined on Sobolev weakly connected spaces, Ukrainian Math. J., 48, 5 (1996), 683-698.

[25] A. KovaleVSKY AND F. NiCOLOSI, On the convergence of solutions of degenerate nonlinear elliptic high order equations, Nonlinear Anal. Ser. A: Theory Methods, 49, 3 (2002), 335-360.

[26] A. A. KovaleVsKiI AND O. A. Rudakova, On the $\Gamma$-compactness of integral functionals with degenerate integrands, Nelinejnye granichnye zadachi, 15 (2005), 149-153.

[27] A. A. KovalevskiI And O. A. Rudakova, On the strong connectedness of weighted Sobolev spaces and the compactness of sequences of their elements, Tr. Inst. Prikl. Mat. Mekh. Nats. Akad. Nauk Ukrainy, 12 (2006), 85-99.

[28] V. A. Marchenko And E. Ya. Khruslov, Homogenization of Partial Differential Equations, Birkhäuser, Boston, 2006.

[29] F. Murat, Sur l'homogeneisation d'inequations elliptiques du 2ème ordre, relatives au convexe $K\left(\psi_{1}, \psi_{2}\right)=\left\{v \in H_{0}^{1}(\Omega) \mid \psi_{1} \leqslant v \leqslant \psi_{2} p\right.$. p. dans $\left.\Omega\right\}$, Publ. Laboratoire d'Analyse Numérique, no. 76013, Univ. Paris VI, 1976.

[30] M. K. V. MuRThy AND G. StAmpaCChiA, Boundary value problems for some degenerate-elliptic operators, Ann. Mat. Pura Appl. (4), 80 (1968), 1-122.

[31] O. A. RudAKOVA, On the coercivity of the integrand of the $\Gamma$-limit functional of a sequence of integral functionals defined on different weighted Sobolev spaces, Tr. Inst. Prikl. Mat. Mekh. Nats. Akad. Nauk Ukrainy, 15 (2007), 171-180.

[32] O. A. Rudakova, On the $\Gamma$-convergence of integral functionals defined on different weighted Sobolev spaces, Ukrain. Mat. Zh., 61, 1 (2009), 99-115.

[33] G. V. SANDRAKOV, On the homogenization of variational inequalities for problems of nonlinear diffusion in perforated domains, Izv. Math., 69, 5 (2005), 1035-1059.

[34] G. V. SANDRAKOV, Homogenization of variational inequalities and of equations defined by a pseudomonotone operator, Sb. Math., 199, 1-2 (2008), 67-98.

[35] I. V. SKRYPNIK AND D. V. LARIN, Principle of additivity in averaging of degenerate nonlinear Dirichlet problems, Ukrainian Math. J., 50, 1 (1998), 136-154.

[36] M. M. VAInBERG, Variational Method and Method of Monotone Operators in the Theory of Nonlinear Equations, Halsted Press, New York-Toronto, 1973.

[37] V.V.ZHikov, Questions of convergence, duality and averaging for functionals of the calculus of variations, Izv. Akad. Nauk SSSR Ser. Mat., 47, 5 (1983), 961-998.

[38] V. V. ZhiKov, Passage to the limit in nonlinear variational problems, Mat. Sb., 183, 8 (1992), 47-84. 